\title{
Filmer les soins. Données vidéo et recherche qualitative
}

\author{
David Pichonnaz, Ph. D.
}

HESAV, Haute école spécialisée de Suisse occidentale, Lausanne, Suisse

Camille Bécherraz, MA

HESAV, Haute école spécialisée de Suisse occidentale, Lausanne, Suisse

Isabelle Knutti, MSc

HESAV, Haute école spécialisée de Suisse occidentale, Lausanne, Suisse

\section{Liliana Staffoni, MSc}

HESAV, Haute école spécialisée de Suisse occidentale, Lausanne, Suisse

Veronika Schoeb, Ph. D.

HESAV, Haute école spécialisée de Suisse occidentale, Lausanne, Suisse

\begin{abstract}
Résumé
Cet article s'intéresse aux enregistrements vidéo comme données de sciences sociales, en particulier dans l'étude des institutions de santé. Sont abordés leurs apports et les enjeux entourant leur production et leur analyse. La vidéo permet la captation de situations naturelles et d'un nombre élevé d'informations. Si cet outil a été principalement utilisé par des études consacrées aux actions situées, il peut être pertinent pour différents types d'études. L'article présente les choix pratiques et les contraintes techniques qu'il implique. Le texte s'intéresse également aux négociations nécessaires afin de faire accepter la présence de caméras au sein
\end{abstract}

Note des auteurs : Les deux projets servant de base à la réflexion présentée dans cet article ont été financés par le Fonds national suisse de la recherche scientifique (subsides 13DPD6_134835 et 100017 159327/1).

RECHERCHES QUALITATIVES - Vol. 36(2), pp. 63-84.

LES ENJEUX ACTUELS DE LA RECHERCHE QUALITATIVE À L'HÔPITAL ET DANS LES INSTITUTIONS DE SOINS DE LONGUE DURÉE

ISSN 1715-8702 - http://www.recherche-qualitative.qc.ca/revue/

(C) 2017 Association pour la recherche qualitative 
des institutions de santé et le degré variable de résistance qui peut y être opposée. Finalement sont abordées les questions éthiques associées à la captation vidéo de données médicales. Au final, l'article souhaite montrer la pertinence de la prise en compte de cet outil de récolte de données parmi ceux étant disponibles aux sciences sociales.

Mots clés

DONNÉES VIDÉO, INSTITUTIONS DE SANTÉ, TRAVAIL, MÉTHODOLOGIE

\section{Introduction}

L'étude des pratiques de travail au sein des institutions (de santé et d'autres domaines) est l'objet d'une longue tradition sociologique: les études pionnières initiées par Hughes (1958) puis Becker et Strauss ${ }^{1}$ à Chicago ont aujourd'hui encore (ou à nouveau) une influence importante sur l'étude des pratiques professionnelles. Nous constatons cependant que les études contemporaines d'inspiration interactionniste ne font pas usage de la vidéo, même lorsqu'elles s'inscrivent dans une approche ethnographique et en dépit de l'importance qui a pu être accordée (au moins par une partie de ces travaux) à l'étude détaillée des pratiques de travail (Avril, Cartier, \& Serre, 2010; Dubar, Tripier, \& Boussard, 2011). C'est davantage du côté de l'ethnométhodologie et de l'analyse de conversation, et plus récemment des workplace studies, que le travail, en particulier soignant, a été scruté au moyen d'enregistrements vidéo. Nous souhaitons montrer dans cet article que ce puissant outil de production de données, qui permet de saisir les pratiques et les interactions dans toute leur épaisseur, mériterait qu'on lui accorde davantage d'intérêt dans les études sur les pratiques soignantes et les institutions de santé.

Cet article s'intéresse dès lors à la vidéo en tant qu'outil de production de données dans les recherches sur les institutions de santé et, plus généralement, en sciences sociales. Nous proposons une analyse des apports, des limites et des enjeux scientifiques, pratiques et éthiques entourant la production et l'usage de données filmées. Notre réflexion ne s'inscrit pas à proprement parler dans le cadre d'une sociologie visuelle: elle aborde la vidéo comme un outil de récolte de données ethnographiques, comme l'enregistrement audio est un outil de captation des interactions verbales classiquement utilisé lors d'entretiens individuels ou collectifs. Notre réflexion est nourrie notamment par deux projets de recherche, l'un terminé et l'autre en cours, au sein desquels l'usage de la vidéo est au centre (voir l'Encadré 1). Le premier est consacré aux interactions entre les soignants et soignantes et les bénéficiaires de soins, le second aux processus de collaboration entre les professionnels et professionnelles. Les deux enquêtes prennent pour terrain d'investigation tant des hôpitaux que des centres de réadaptation ou des institutions médico-sociales. 
Nous pouvons nous appuyer, dans la réflexion présentée ici, sur une double expérience de conduite de projets de recherches nationaux financés par le Fonds national suisse de la recherche scientifique et ayant recours à des enregistrements vidéo comme données principales.

Le premier projet, intitulé Discharge planning in rehabilitation centers : What about patient participation? avait pour objectif de mettre en lumière les stratégies communicationnelles mises en place par les professionnels et professionnelles de santé et par les patients et patientes dans le cadre de réunions interdisciplinaires visant la planification de sortie de ces derniers. Dans le cadre de ce projet, nous avons recruté 37 bénéficiaires de soins au sein de trois institutions différentes et avons filmé 164 réunions ou visites (médicales ou infirmières), correspondant à 25 heures d'enregistrements vidéo. Les analyses montrent que les professionnels et professionnelles s'appuient sur des ressources communicationnelles variées (langage verbal, gestes, regards, documents) et utilisent des stratégies diverses pour faire participer les patients et patientes lors des réunions ou des visites cliniques.

Le second projet, en cours, est intitulé Interprofessional collaboration: How do health professionals interact with each other in collaborative practice situations. Il vise à mieux comprendre où, quand et comment les praticiens et praticiennes appartenant à différentes professions de la santé (et dans certains cas du social) collaborent au sein d'institutions de santé de natures diverses. Le cœur du projet consiste à investiguer la manière dont se déroule la collaboration « en train de se faire », avec pour objectif d'observer comment les professionnels et professionnelles gèrent les exigences de la pratique collaborative et d'identifier les ressources, à la fois verbales et non verbales, sur lesquelles ils et elles s'appuient pour collaborer. Cette analyse est basée sur des enregistrements vidéo de situations de pratiques collaboratives variées (réunions, visites cliniques, cothérapies, discussions informelles).

La démarche de recherche adoptée dans le cadre de ces deux projets, fortement ancrée dans la tradition qualitative, est en partie inspirée de l'analyse conversationnelle.

Encadré 1. Deux projets recourant aux enregistrements vidéo comme données principales.

Cet article propose une analyse en deux temps : premièrement sont abordés les usages et les apports de la vidéo dans la recherche de sciences sociales, ainsi que les enjeux scientifiques et pratiques l'entourant. Deuxièmement, nous rendons compte de la manière dont on peut « faire entrer » des caméras au sein des institutions de soins et des raisons pouvant expliquer les plus ou moins grandes difficultés rencontrées lors de 
la négociation de l'accès au terrain. Cette partie s'intéresse également aux questions éthiques associées à la captation vidéo de données médicales.

\section{Les avantages méthodologiques et les apports scientifiques de la vidéo}

Comparée aux autres outils de production de données en sciences sociales, la vidéo a pour spécificités principales de permettre l'enregistrement de situations naturelles (contrairement à l'entretien) et de capter un degré élevé d'informations associant sons, images et mouvements (contrairement au carnet d'observation) : «Tous les éléments constituant la signification d'une image sont présents simultanément $»^{2}$ [traduction libre] (Raab \& Tänzler, 2012, p. 86). Les enregistrements vidéo offrent ainsi la possibilité de conserver durablement les interactions de tous types, et ce, dans toute leur consistance et leur complexité. La durée des séquences filmées, bien que fortement variable, peut certes apparaître courte comparée à la présence longue de l'ethnographe sur son terrain. L'usage de la vidéo n'exclut cependant pas l'observation directe des pratiques par immersion, et, plus encore, elle doit être conçue comme un outil de récolte de données dont la densité compense en quelque sorte la durée (Knoblauch, 2012). Comme le relèvent en effet Knoblauch, Soeffner, Raab et Schnettler (2012), « les données vidéo font sans nul doute partie des données les plus complexes dans la recherche empirique en sciences sociales $»^{3}$ [traduction libre] (p. 14). Comme l'enregistrement d'un entretien, mais avec la naturalité et la densité qui lui sont propres, la vidéo a en outre pour caractéristique extrêmement avantageuse sa transférabilité, contrairement aux notes de terrain. Elle «permet en effet de détacher le moment de l'observation du déroulement de la situation observée, et cette distance rend possible une exploration répétée du document, ainsi que la découverte de nouvelles caractéristiques autrement inaperçues » (Lambelet, 2010, p. 4). Pouvant être partagée avec d'autres chercheurs et chercheuses, mais également avec les milieux filmés, elle permet non seulement une analyse approfondie des pratiques, mais favorise également l'analyse collective des données, les possibilités d'archivage et de création de bases de données, ainsi que - après sélection d'extraits - leur diffusion aux actrices et acteurs étudiés ou dans le cadre de présentations orales (la publication en format papier des données vidéo pouvant en revanche poser des problèmes spécifiques). Davantage que les notes ethnographiques, l'outil offre une forme de transparence, en ce qu'il permet de diffuser des extraits plus bruts des actions observées.

\section{Capter le langage verbal et les mouvements du corps}

Les données récoltées par vidéo sont denses, car elles enregistrent dans les détails trois dimensions constitutives des comportements humains : le langage verbal, les pratiques non discursives et la communication non verbale, ainsi que les rapports entre humains et objets. Comme on peut le faire avec un enregistrement audio d'entretiens - mais non en se basant sur un carnet de notes ethnographiques -, le langage verbal peut être, grâce à la vidéo, saisi dans toute sa complexité et ses nuances. L'analyse peut ainsi être 
attentive au choix des mots et à la tournure des phrases construites par les protagonistes étudiés, aux intonations, aux silences, à la manière dont les personnes filmées commencent ou terminent une conversation, aux tours de paroles, à la dynamique de groupe, pour ne donner que quelques exemples. En ce qui concerne les pratiques et la communication non verbales, la vidéo offre l'avantage immense de pouvoir capter les positionnements et les mouvements des corps, les gestes, les expressions du visage, ou encore l'orientation des regards. La plupart de ces éléments constituent des ressorts essentiels des interactions humaines, mais restent pourtant largement sous-évalués par les sciences sociales. Comme le relève Parry (2010), les soins en particulier sont souvent délivrés dans le cadre d'interactions en face à face et consistent en discours et pratiques impliquant le corps, ce dernier constituant dès lors un enjeu central dans l'analyse de ce type d'activité. Des travaux montrent par exemple que le regard et la posture corporelle des professionnels et professionnelles ont un impact sur la fluidité du discours des bénéficiaires de soins (Ruusuvuori, 2001; Tiitinen \& Ruusuvuori, 2012) et sur la collaboration entre ces deux catégories d'individus (Robinson, 1998). D'autres ont pu étudier les instructions tacites données par les chirurgiens à celles et ceux qui les assistent, basées sur des gestes situés (Mondada, 2014) ou la manière dont, en anesthésie, la coordination des activités entre les professionnels et professionnelles consiste notamment à anticiper les gestes des uns et des autres (voir le concept d'intercorporeal knowing, Hindmarsh \& Pilnick, 2007). L'enregistrement vidéo permet finalement d'observer les relations entre êtres humains et objets matériels, en particulier l'usage d'outils, des plus simples (documents textuels par exemple) aux plus complexes, aux technologies les plus pointues. La vidéo permet ainsi de tenir compte davantage de l'environnement matériel des interactions humaines (donc également de l'agencement spatial) et de la manière dont il est utilisé et forme contexte pour l'action. Keel et Schoeb ont par exemple pu montrer l'importance jouée par les documents écrits dans les interactions entre professionnels et professionnelles lorsqu'il s'agit d'accomplir une transition entre différentes phases lors de réunions d'équipe (Keel \& Schoeb, 2016).

\section{Des affinités avec certaines approches de sciences sociales?}

Au sein des sciences sociales, les usages les plus courants de la vidéo se rencontrent sans nul doute du côté de l'ethnométhodologie et, plus encore, de l'analyse de conversation (Parry, 2010). Cet outil attire également, de manière plus générale, les chercheuses et chercheurs intéressés par les logiques interactionnelles et par les interactions entre humains et non-humains, et est le plus souvent associé aux approches les plus inductives (Schubert, 2012). Nous pensons cependant que la vidéo, en particulier si elle est combinée à d'autres outils, peut être d'un usage pertinent dans le cadre de la plupart des études de sciences sociales, en particulier celles étant les plus inspirées de l'ethnographie. En dépit des contraintes techniques qui pèsent sur son usage, et pour peu que le détail des interactions constitue au moins en partie l'objet de 
la recherche, il nous semble en effet que cet outil a toute sa place dans l'arsenal méthodologique des sciences sociales.

Les recherches inspirées de l'ethnométhodologie et de l'analyse de conversation, et toutes celles concentrées sur les pratiques sociales, possèdent des accointances particulières avec la vidéo dans la mesure où elles «mettent la priorité sur l'accomplissement situé et interactionnel des actions pratiques $»^{4}$ [traduction libre] (Heath, Hindmarsh, \& Luff, 2010, p. 1). Ces approches sont en particulier intéressées par la structure temporelle et séquentielle des interactions que la vidéo permet de capter minutieusement (Luckmann, 2012). Dans cette optique, elle permet notamment d'étudier la manière dont les technologies et les outils sont utilisés séquentiellement dans les activités de travail, la façon dont les acteurs et actrices constituent le sens de leur activité en situation, ou encore comment s'organise le travail en équipe (Heath et al., 2010). L'outil est indispensable à celles et ceux qui souhaitent observer comment les individus coordonnent leurs actions en regardant ce que font les autres et en rendant visibles ou disponibles certaines de leurs actions, ou la manière dont les interactions sont dépendantes de pratiques non discursives, comme la nécessité de remplir des formulaires, par exemple. Dans cette perspective, les workplace studies, ou plus généralement les approches s'intéressant aux activités sur la place de travail et à leur organisation située ont fait un usage extensif de la vidéo, souvent pour concentrer leur regard sur l'usage des technologies, en particulier informatiques (Luff, Hindmarsh, \& Heath, 2000).

De manière plus générale, la vidéo a été utilisée dans le cadre d'études de sciences sociales plaçant au centre de leurs analyses la perspective des personnes étudiées et portant leur intérêt sur les ressources sur lesquelles elles peuvent s'appuyer pour accomplir leurs activités et actions quotidiennes. En se basant sur la sociologie de Berger et Luckmann, des chercheurs et chercheuses ont pu ainsi parler d' «herméneutique vidéo », en étant attentifs à la manifestation, ainsi filmée, des perceptions et interprétations que les acteurs et actrices effectuent à propos de la réalité qui les entoure (Raab \& Tänzler, 2012). Plus généralement, ce type de données est utile à toutes celles et tous ceux qui analysent les «structures, schémas d'interaction, tels que la coordination des activités de travail, le cours des conflits familiaux ou des réunions de travail $»^{5}$ [traduction libre] (Knoblauch, 2012, p. 72; voir aussi Lambelet, 2010). En parallèle, les approches cliniques de l'activité développées en particulier par Yves Clot et son équipe (Clot, Faïta, Fernandez, \& Scheller, 2000), plus proche de la psychologie, font également un usage important de la vidéo. Les enregistrements sont dans ce cas montrés aux personnes filmées, dans une démarche d' "autoconfrontation croisée » dans le cadre de laquelle le visionnement de l'activité professionnelle par celles et ceux qui l'accomplissent, en compagnie des équipes de recherche, permet de faire émerger les « impensés » des pratiques, dans un but clinique. 
Dans le débat entre sociologie historique et structurale d'un côté, interactionniste et l'on pourrait dire «situationnaliste» de l'autre, la vidéo est donc, jusqu'à aujourd'hui en tous cas, clairement un outil privilégié par les secondes. Combinée avec des observations ou des entretiens, elle peut pourtant être utile à des travaux et des approches qui tiennent compte d'éléments non observables dans les situations filmées, que ce soit le contexte organisationnel, les socialisations individuelles et tous les facteurs associés à la structure sociale et au contexte historique. Les données vidéo, comme le fait remarquer Schubert (2012), peuvent être considérées comme un moyen de concentrer son attention sur un type de situations en particulier, alors que l'ethnographie insiste sur l'ouverture du regard. Le travail ethnographique, qu'il se base sur des observations ou des entretiens approfondis, doit cependant permettre de déterminer et de circonscrire les situations ou les actions qu'il est pertinent d'enregistrer grâce à la vidéo. Ce sont dès lors davantage les questions et les hypothèses de recherche qui doivent selon nous présider au choix d'avoir recours ou non à la vidéo (et à la manière de s'en servir) que l'école de pensée dans laquelle s'inscrit l'étude.

\section{Les choix à opérer : entre théorie et contraintes techniques}

L'opérationnalisation d'une démarche de recherche faisant usage de la vidéo pose cependant à chaque fois la question de déterminer, d'une part, quelles situations doivent être filmées et, d'autre part, la manière dont elles doivent l'être. Si ces choix doivent bien entendu, comme tout choix méthodologique, découler des questions de recherche et des hypothèses, ils n'en demeurent pas moins contraints par des enjeux techniques étant spécifiques à la vidéo, en particulier le positionnement de la caméra, le choix du cadrage et le type de prise de son.

En plus du processus intellectuel de construction de l'objet de recherche, la sélection des situations filmées se doit de bénéficier de données récoltées préalablement, que ce soit des entretiens (collectifs ou individuels) ou des observations directes. La connaissance de l'organisation et du fonctionnement des institutions de santé est en effet un préalable essentiel pour déterminer les situations au sein desquelles se jouent les enjeux constituant l'objet de recherche. En ce qui concerne l'étude des phénomènes de collaboration par exemple, l'on peut décider de filmer des réunions entre professionnels et professionnelles, ce que nous avons largement fait dans le cadre de nos deux projets de recherche (voir l'Encadré 1). Dans ce cas, l'on procède comme on filmerait un entretien collectif dans le but de pouvoir ensuite transcrire précisément la manière dont les acteurs et actrices parlent des patientes et des patients, comment est négocié le choix des cas dont on parle, quelles informations sont échangées, comment se forme et évolue la dynamique de groupe et la manière dont sont prises les décisions. Les séquences filmées permettent également de porter attention au langage non verbal, en particulier à l'orientation des regards. Les 
entretiens et périodes d'observations préliminaires ont permis non seulement d'identifier les réunions les plus pertinentes à filmer, mais également d'autres moments de collaboration moins formalisés, mais mettant tout autant en relief - sinon plus - les enjeux et problèmes posés par la collaboration interprofessionnelle: discussions informelles dans les couloirs ou d'autres lieux spécifiques, thérapies prodiguées simultanément par plusieurs professionnels et professionnelles sur un patient ou une patiente, formation d'infirmiers et d'infirmières à certains gestes par des thérapeutes. Identifier ces situations afin de les filmer n'est possible qu'à condition d'avoir une connaissance fine de l'institution étudiée. L'on peut imaginer un autre type de dispositif méthodologique, dans le cadre duquel le recours à l'enregistrement vidéo est moins central, mais où il permettrait de capter des situations considérées comme paradigmatiques, ou illustratives de certains phénomènes analysés par ailleurs grâce à une observation de type ethnographique (préalable ou parallèle). Dans ce cas, la caméra permet d'enregistrer les pratiques et interactions dans toute leur épaisseur, pour appuyer et compléter l'analyse basée sur l'observation, et ce, au moyen de transcriptions plus détaillées que les notes de terrain.

Une fois les situations identifiées, il s'agit de déterminer la manière dont elles vont être filmées, ce qui soulève une série de questions pratiques et techniques associées à des enjeux scientifiques complexes, dont la plupart sont communs à toute étude ethnographique. Celle d'abord de la position de la caméra, l'idée étant le plus souvent de tenter de rendre sa présence la plus discrète et la moins gênante possible, par exemple en privilégiant la proximité d'un mur ou le coin d'une pièce. Une caméra fixe, immobile et positionnée sur un trépied, imite d'une certaine manière un regard observateur: discrète, elle permet de limiter les risques de manquer une partie de l'action. Cette position est en particulier bien adaptée lorsque le nombre de personnes filmées n'est pas trop important, que les discussions que ces personnes peuvent mener en parallèle sont également limitées et que l'activité filmée ne déborde pas dans d'autres lieux. Elle est bien ajustée par exemple à l'enregistrement vidéo d'une réunion, d'une consultation ou d'une situation d'enseignement. L'on peut cependant opter, au contraire, pour une caméra mobile. Prise en mains par le chercheur ou la chercheuse, la caméra peut ainsi suivre ce que les acteurs et actrices sont en train de faire, en étant au plus proche de l'action. Si cette manière de procéder rend plus difficile l'analyse de la structure de l'action (les perspectives proches de l'analyse de conversation privilégient dès lors le plus souvent la caméra fixe), une caméra mobile suit l'œil du chercheur ou de la chercheuse (y compris grâce au zoom), mettant en lumière certains éléments en en cachant d'autres. Cette manière de procéder permet de suivre les protagonistes, par exemple s'ils se retirent pour téléphoner ou lorsqu'ils se déplacent dans le cadre de thérapies physiques. Elle permet également de capter des gestes précis ou la manipulation d'objets de petite taille. Lorsque ce sont les gestes précis des individus qui constituent l'objet d'analyse, la caméra mobile peut en effet 
être la solution la plus adaptée, comme le relève Schubert (2012), relatant une recherche à propos du travail en salle d'opération. Alors qu'il avait initialement prévu de recourir à des caméras fixes, la caméra mobile s'est avérée dans les faits nécessaire, le corps des professionnels et professionnelles cachant le plus souvent leurs gestes aux caméras fixes, positionnées proches des murs pour ne pas gêner l'action. Cet usage mobile de la caméra est particulièrement adapté lorsque l'analyse porte sur des gestes courts, délimités dans le temps et non accompagnés de dialogues, par exemple lorsqu'il s'agit d'étudier la collaboration muette entre deux professionnels ou professionnelles lors d'une intubation (Schubert, 2012). Le choix entre caméra fixe ou mobile n'est pas exclusif : rien n'empêche en effet de faire usage de plusieurs caméras, dont l'une peut être mobile. Deux caméras fixes peuvent également être utiles lorsque plusieurs activités sont accomplies simultanément sur le même lieu, si les personnes filmées tiennent plusieurs discussions en parallèle, ou encore pour filmer les documents ou les écrans dont elles se servent. Dans leur étude sur la collaboration dans le cadre de la chirurgie endoscopique, Koschmann, LeBaron, Goodwin, Zemel et Dunnington (2007) ont ainsi utilisé les images intracorporelles filmées par l'équipe chirurgicale, ainsi que deux caméras : l'une fixe, enregistrant l'ensemble de ce qui se passe dans la pièce, l'autre, de taille réduite et placée sur la tête du chirurgien, permettant de filmer précisément ses gestes.

Que la ou les caméras soient fixes ou mobiles, il s'agit en effet de choisir également ce qui est introduit dans le cadre et ce qui reste en dehors de celui-ci. D'un point de vue technique, cela consiste à sélectionner l'angle (ou les angles) de la caméra, y compris la taille du zoom. Un angle grand fait prendre le risque de perdre des détails, par exemple des gestes, des regards, des expressions faciales ou la manipulation d'objets de petite taille. Un angle trop serré empêche quant à lui de saisir des actions coordonnées, en ôtant la possibilité d'avoir une vue d'ensemble sur ce qui se déroule dans une situation donnée. Ce sont ici les questions et hypothèses de recherche qui doivent guider le choix de l'angle de prise de vue, comme lorsque l'on constitue un échantillon d'interviewés et interviewées ou que l'on choisit d'orienter son regard d'ethnographe sur certaines situations et pas d'autres. En matière d'enregistrements vidéo, les possibilités techniques disponibles aujourd'hui permettent cependant (en partie) de renoncer à devoir opérer certains choix : lorsque les images sont de bonne qualité, l'on pourra faire usage d'un zoom numérique lors du visionnement ultérieur, afin d'observer certains détails. Il est également devenu facile techniquement, si l'on a utilisé deux caméras, de juxtaposer ensuite les images afin d'analyser une même situation en l'observant sur plusieurs angles simultanément.

Finalement, la question de l'enregistrement sonore oblige également à faire des choix techniques. Les microphones intégrés aux caméras ne suffisent généralement pas à enregistrer des conversations entre individus s'ils ne sont pas à proximité immédiate de ceux-ci. Les choses se compliquent encore lorsque plusieurs discussions sont 
menées en parallèle. Un microphone de qualité, installé sur la caméra, peut être un palliatif. Il peut également être déposé à proximité des personnes filmées, avec cependant le défaut de rendre le dispositif d'enregistrement plus visible à leurs yeux. Avec le même risque, l'on peut également, en particulier pour les interactions n'impliquant que deux individus, utiliser un micro-cravate fixé sur l'une des deux personnes. Cette solution est également potentiellement pertinente lorsque les personnes filmées se déplacent souvent.

\section{Comment faire accepter la caméra? Stratégies de recherche et caractéristiques des terrains}

Notons d'emblée que l'usage de la vidéo est largement diffusé dans le monde de la santé. L'usage de la caméra n'apparaît donc pas étranger de prime abord aux individus étudiés, contrairement aux outils classiques de sciences sociales que sont l'entretien de recherche ou les méthodes ethnographiques ${ }^{6}$. Dans la plupart des institutions, des enregistrements sont régulièrement effectués : sont par exemple filmés des patients et patientes dans le but d'analyser leurs troubles lors de déplacements à la marche, ou des professionnels et professionnelles (en interaction ou non avec les bénéficiaires de soins) afin d'analyser ou d'améliorer les pratiques (par exemple dans le cadre de supervisions avec un ou une psychiatre). Ces séquences, tournées par les sujets dans le cadre de leurs pratiques quotidiennes, ont donc des visées pédagogiques ou réflexives, mais elles peuvent également être utilisées à des fins de recherche. Elles sont courantes en particulier dans le domaine de la réhabilitation, où l'évaluation de l'état de santé des patients et patientes repose largement sur une observation de ceux-ci lors d'activités fonctionnelles, en psychiatrie également, où les consultations sont souvent filmées à des fins d'analyse et d'évaluations, ainsi qu'en médecine du sport ou en pédiatrie. Cette diffusion de l'usage des images ne garantit cependant pas, tant s'en faut, une acceptation de la vidéo, dans ces domaines comme dans les autres, lors des négociations d'accès aux terrains d'enquête, mais fait de cet outil un objet relativement familier.

La vidéo exacerbe cependant, comme le relèvent Heath, Hindmarsh et Luff (2010), certaines des difficultés liées à l'accès et aux négociations d'entrée sur le terrain propres à toute enquête qualitative réalisée par immersion (Beaud \& Weber, 2010; Darmon, 2005). Dans les différents terrains que ces auteurs ont étudiés en faisant usage de la vidéo, ils déclarent cependant avoir rencontré peu de résistances, tant à l'hôpital que dans d'autres contextes professionnels, à condition d'être sensibles aux demandes des institutions et de tenir compte des préoccupations des participants et participantes (Heath et al., 2010). Nous faisons le même constat: sur plus de quinze institutions ou unités contactées, deux seulement ont refusé que nous conduisions des enregistrements vidéo de situations de travail en leur sein. En revanche, les difficultés de négociation de l'accès au terrain, les types de contreparties exigées, l'ampleur de 
l'acceptation de la caméra ou encore le temps de présence sur place accordé ont grandement varié d'une institution à l'autre.

Lorsque l'on s'intéresse à l'acceptation de la caméra au sein d'une institution, et plus généralement à la négociation de l'accès aux terrains, il est indispensable de distinguer les négociations conduites avec la direction et les cadres, que nous traitons d'abord en deux temps (dans les sections Stratégies d'accès au terrain et Terrains ouverts, terrains fermés), de l'obtention du consentement individuel de chaque professionnelle ou professionnel à être filmé, que nous évoquerons ensuite (section Récolter les consentements individuels des professionnels et professionnelles). Les personnes avec lesquelles l'on négocie l'accès sont en effet rarement celles que l'on filme ensuite (Heath et al., 2010). La question délicate du consentement des patients et patientes, qui est entourée d'enjeux éthiques spécifiques, est traitée dans la dernière section (Faire signer le «consentement» aux patients et patientes : la gestion des normes et contraintes éthiques).

\section{Stratégies d'accès au terrain : négocier avec la direction}

Multiples sont les stratégies qui peuvent être mises en place pour faire accepter l'usage de la vidéo auprès des instances dirigeantes d'une institution de santé, ou plus largement la présence des chercheurs et chercheuses au sein de leurs services. La première consiste à anticiper les intérêts que les institutions peuvent y trouver. Notre focalisation sur la collaboration, tant entre les soignants et soignantes et les bénéficiaires de soins qu'entre professionnels et professionnelles, correspond à des préoccupations majeures dans le monde de la santé contemporain. Cherchant en outre à étudier des lieux où la collaboration est développée ou fait l'objet d'une préoccupation particulière, nous avons maximisé nos chances de susciter un intérêt de la part des diverses personnes contactées. Il s'agit d'un choix scientifique - étudier la collaboration "en train de se faire » et non pas produire une étude supplémentaire mettant en évidence les difficultés de la collaboration - ayant cependant des conséquences méthodologiques en l'occurrence positives. Notre démarche a souvent consisté à présenter d'abord le projet lors de réunions de cadres, ou parfois à la direction uniquement. Une attention particulière a été apportée à présenter les projets comme n'ayant pas de visées évaluatives. En insistant sur les connaissances qu'ils permettent de produire à propos des interactions sociales, nous avons pu nous écarter d'une étude qui viserait par exemple à mesurer le degré de collaboration, sa qualité ou encore le respect des guidelines officielles ou des recommandations des associations professionnelles. Par ailleurs, nous avons constaté l'importance de pouvoir compter sur la légitimité de l'organe de financement, le Fonds national suisse de la recherche scientifique, connu des acteurs et actrices puisqu'il finance également des recherches dans le domaine de la médecine. Associée au fait que nous avons conduit deux projets consécutifs et qu'une partie importante de l'équipe est issue du monde des soins ${ }^{7}$, cette 
institution de financement permet de construire une professionnalité de chercheurs et chercheuses, en dépit du caractère qualitatif de notre approche, dans un milieu habitué aux approches quantitatives.

Parmi les stratégies adoptées, une autre consiste à prendre au sérieux les préoccupations spécifiques des institutions contactées, notamment les situations leur posant problème en termes de collaboration. Dans une institution de taille importante, réunissant un grand nombre de professions différentes, nous avions par exemple l'intention d'organiser et de filmer un ou deux entretiens collectifs consacrés à la collaboration interprofessionnelle en général (préalablement à l'enregistrement vidéo de situations naturelles) réunissant un porte-parole par métier, choisi parmi les professionnels et professionnelles de terrain, selon la procédure que nous avions mise en œuvre ailleurs. En réponse, la direction nous a proposé de réunir des groupes plus restreints, ne visant pas l'exhaustivité des professions pour chaque entretien, mais autour de domaines spécifiques de prise en charge interdisciplinaire faisant débat au sein de l'institution - à propos desquels la direction éprouve de l'intérêt pour nos analyses. Si l'on part du principe que ce qui préoccupe les personnes étudiées mérite d'être étudié, à condition que la manière de le questionner reste du ressort des chercheuses et chercheurs, cette situation est alors de type gagnant-gagnant. Sinon, elle peut être acceptée en contrepartie de la possibilité de filmer d'autres situations de travail ultérieurement.

Afin de minimiser l'impact de la caméra, d'autres stratégies peuvent également être mises en place, telles que l'installation d'une caméra fixe, actionnée par les personnes étudiées, dans un lieu particulier (salle de réunion, cabinet de consultation) ou le fait de leur confier une caméra afin qu'elles filment elles-mêmes les situations qu'elles jugent intéressantes. Cette seconde stratégie permet de produire des données supplémentaires, puisque les choix opérés par les professionnels et professionnelles peuvent être analysés en tant que tels : « lorsque le document [audiovisuel] est produit par les acteurs eux-mêmes, il peut nous livrer les représentations, les cadrages et les focales privilégiés des participants » (Lambelet, 2010, p. 4).

Finalement, il faut noter que notre démarche de recherche inclut une phase de restitution, lors de laquelle les résultats de recherche sont présentés et discutés avec les individus ayant participé à l'étude. Or les projections de séquences vidéo, davantage que les présentations orales classiques, permettent plus facilement d'organiser des ateliers de discussion ${ }^{8}$. Il est à noter que ces ateliers ne visent pas la production de catalogues de bonnes pratiques, mais à stimuler une réflexion collective sur la manière d'améliorer, par exemple, la participation des patients et patientes aux décisions les concernant. Lors de la phase de restitution de notre projet consacré à ce thème, les ateliers ont été jugés positivement par les participants et participantes. Certains ont explicitement mentionné leur intérêt pour les séquences vidéo. La possibilité de 
visionner plusieurs fois les enregistrements sélectionnés par les chercheurs et chercheuses afin de décrypter en détail les processus de communication a en particulier été appréciée (Schoeb, Hartmeier, \& Keel, 2015). Le fait de pouvoir proposer ces ateliers dès le début des négociations contribue à faire accepter la vidéo, puisqu'elle facilite la promesse d'un retour auprès des personnes impliquées. Dans ce cadre, le fait de travailler sur plusieurs terrains est un atout important: les comparaisons entre institutions que nous pouvons promettre, notamment à propos de dispositifs organisationnels, rencontrent souvent l'intérêt des instances dirigeantes.

Terrains ouverts, terrains fermés : rapport à la recherche et position sociale des institutions de santé

Si l'on s'intéresse aux facteurs pouvant favoriser l'acceptation de la caméra par les responsables des institutions étudiées, et plus largement rendant plus probable la participation du personnel des organisations de santé à une étude qualitative, quatre spécificités propres aux institutions ou unités visées nous paraissent essentielles. Il s'agit de leur degré d'intérêt pour la thématique de la recherche, que nous avons évoqué dans la section précédente, de leur rapport à la recherche scientifique en général, de leur volonté ou non d'être visible dans le champ scientifique international ainsi que de leur degré de reconnaissance et de légitimité dans le monde de la santé en Suisse.

Les négociations ont été les plus difficiles, conduisant parfois à des échecs, avec les unités activement engagées dans la recherche biomédicale, subissant une pression à la publication et occupant une position reconnue dans le monde de la santé en Suisse ou de pouvoir au sein de leur institution. Ce sont en particulier les unités de soins aigus au sein des hôpitaux universitaires, et notre expérience montre que les difficultés sont plus grandes lorsque ces unités sont actives dans un domaine médicalement valorisé (par exemple la neurologie) ou qu'elles occupent une position de pouvoir au sein de leur institution d'appartenance en raison de leur taille. Les responsables de ces unités ont mis en avant leur expertise en matière de recherche scientifique, par exemple en exhibant le nombre de publications produites annuellement par leurs services ou, pour l'un d'entre eux en particulier, commentant la qualité de notre projet de recherche et relevant des défauts selon lui essentiels. Ces types de responsables, avec lesquels les négociations ont été plus difficiles et n'ont pas toujours pu aboutir, nous ont réclamé de faire figurer leurs noms sur un article publié dans le cadre de notre recherche?

Dans un autre service de soins pourtant aigus et au sein d'un hôpital également universitaire, nous avons rencontré des difficultés bien moindres : les négociations ont été simples et rapides, aucune contrepartie n'a été exigée et la présence de la caméra a été acceptée dans la plupart des situations de travail. Le fait que cette unité soit de taille réduite, qu'elle soit largement moins active dans le domaine de la recherche et qu'elle soit dès lors moins contrainte à produire des publications nous semble expliquer en 
partie cette facilité. Par ailleurs, s'agissant d'un domaine de soins moins reconnu au sein de l'institution, la volonté de le rendre visible a largement joué en notre faveur ${ }^{10}$. Nous avons pu par exemple le constater lorsque, répondant positivement à une demande d'enregistrement vidéo d'une réunion interdisciplinaire, le directeur a déclaré qu'il s'agissait d'un moyen pour eux de «faire comprendre» les contraintes et les spécificités de leur activité.

Notre expérience montre cependant que ce sont les institutions actives dans des domaines moins reconnus dans la hiérarchie médicale ou n'ayant pas d'attaches universitaires qui présentent le plus grand degré d'ouverture à la recherche qualitative de sciences sociales. L'accueil a, en effet, été facile à négocier dans des institutions de soins chroniques ou de réhabilitation. Cela découle, d'une part, d'un intérêt plus marqué par ces services ou institutions pour les questions de collaboration interprofessionnelle. D'autre part, cela n'est pas étranger à leurs positions spécifiques dans le monde de la santé, celles-ci ne les poussant pas à exiger des contreparties et les rendant plus sensibles à ce que notre regard extérieur se pose sur elles.

Il faut à ce sujet évoquer une institution de soins de réhabilitation au sein de laquelle nous avons pu bénéficier d'un accueil particulièrement ouvert. Bien qu'il s'agisse d'un hôpital régional subissant peu de pression à produire de nouvelles connaissances scientifiques, cette institution se trouve dans une phase d'agrandissement et de rapprochement avec un hôpital universitaire. Elle suit donc une stratégie de renforcement de sa position dans le monde de la santé en Suisse. Pourtant, toutes les portes nous y ont été ouvertes avec un enthousiasme réjouissant. L'appui de la direction dans les démarches de recherche de consentement des professionnels et professionnelles et des bénéficiaires de soins à être filmés a été d'une aide précieuse. Nous avons pu présenter notre recherche et répondre aux questions des collaboratrices et des collaborateurs lors d'une assemblée en plénum. La liste des noms et adresses courriel de tout le personnel nous a par ailleurs été fournie, ainsi que, chaque semaine, la planification des types de thérapies qui nous intéressaient. Les soignants et soignantes responsables de l'admission des nouveaux patients et patientes se sont chargés de les informer de notre présence et nous ont épaulés dans la récolte de consentements à être filmés. Il nous semble que cette ouverture découle d'un contexte interne favorable, en raison d'un intérêt pour notre objet de recherche, mais également d'un rapport à la recherche et d'une position dans le monde de la santé spécifiques. Si peu de recherches sont aujourd'hui produites au sein de cette institution, elle a en effet un intérêt à en conduire davantage, en raison de sa stratégie d'expansion. Les discussions que nous avons eues avec la direction montrent clairement que, dans ce contexte, la collaboration avec notre équipe est perçue comme un moyen de rendre visible une activité de recherche. En outre, la direction est engagée dans un processus de développement de l'interdisciplinarité, dont elle estime qu'il est incomplet. Ouverte et intéressée par la critique, elle trouve un intérêt à ce que des chercheuses et 
chercheurs extérieurs à l'institution contribuent à la réflexion interne à propos de cet enjeu majeur. En outre, le recours à la vidéo pour analyser des moments de collaboration interprofessionnelle avait été envisagé par le directeur, qui a trouvé avec notre projet une possibilité de déléguer cette pratique ${ }^{11}$.

\section{Récolter les consentements individuels des professionnels et professionnelles}

Une fois les négociations avec la direction abouties, il faut encore faire accepter la caméra aux collaboratrices et collaborateurs de tous rangs. Parmi les stratégies adoptées, la plus importante consiste à suivre des étapes préalables avant d'introduire la caméra. Aux premiers rendez-vous avec la direction puis les cadres succède la conduite d'un entretien collectif avec des membres du personnel. La confiance ainsi gagnée, l'étape suivante consiste en une période d'observation directe sans usage de la caméra, pour finaliser l'établissement de liens de confiance et déboucher enfin sur la captation vidéo. C'est également l'occasion de discuter des objectifs et du déroulement des enregistrements, afin de répondre aux préoccupations des personnes qui seront potentiellement filmées. La possibilité de se rétracter après avoir été filmé, et donc l'effacement possible des séquences, a toujours été offerte, mais jamais utilisée par les professionnels et professionnelles. Certaines actrices et certains acteurs ont en revanche accepté d'être enregistrés (son), mais pas filmés (image). Cela peut constituer une alternative, s'il est possible qu'ils restent hors-champ, et à condition que le langage non verbal ne soit pas au cœur de l'étude. Une attention particulière doit par ailleurs être portée à se distinguer des démarches d'évaluation de leur "qualité », par des inspectrices et inspecteurs extérieurs, dont font l'objet les institutions de santé. Une difficulté importante à surmonter, en particulier dans les institutions de soins aigus, est la charge de travail et le stress engendré par l'urgence des situations de travail qui rendent parfois difficile l'établissement de contacts informels « de couloir», dont l'importance dans le cadre d'une enquête de terrain est pourtant bien connue. Il est en outre important de ne pas mettre les personnes filmées devant le fait accompli, en les prévenant plusieurs jours en avance de la présence de la caméra. Cependant, la planification serrée du travail, les absences régulières et les imprévus découlant des admissions ou de l'état de santé des patients et patientes rendent la planification des enregistrements très difficile dans certaines institutions. Dans les plus grandes en particulier, malgré des courriels envoyés aux professionnelles et professionnels censés être présents lors des situations filmées et aux responsables d'équipe, il arrive souvent que les personnes présentes ne soient pas celles initialement prévues, ce qui peut provoquer des plaintes de leur part au moment où il s'agit de procéder aux enregistrements vidéo, ainsi qu'une difficulté pratique à faire signer, en cas d'acceptation, les documents de consentement, car cela doit alors se faire dans l'urgence. La présence longue permet d'y remédier, comme elle permet d'ailleurs de pallier la méfiance des participants et participantes dans le cadre d'observations ethnographiques (Foley, 2016), de même que la flexibilité des chercheurs et 
chercheuses dans la planification de la présence sur place. Prévenir suffisamment en avance de la présence de la caméra permet par ailleurs aux professionnels et professionnelles qui ne souhaitent pas être filmés, dès lors souvent plus attentifs à nos courriels, de se faire remplacer ou de s'arranger pour ne pas être présents lors des situations que nous avons prévu de filmer. Cela n'est cependant pas toujours possible, les relations hiérarchiques pouvant alors s'immiscer dans la négociation $d u$ consentement des personnes, comme le montre l'exemple présenté ci-après. Nous étions alors en train de filmer une réunion interdisciplinaire lorsqu'un médecin assistant (ayant récemment rejoint le service) entre dans la salle. S'ensuivent les interactions suivantes :

Enquêteur (assis au fond de la salle, s'approche du médecin assistant et s'adresse à lui, ce qui interrompt le cours de la réunion) : "Est-ce qu'on vous a prévenu que la séance était filmée dans le cadre de notre recherche sur la collaboration interprofessionnelle?»

Médecin cadre (répondant à sa place) : «J'ai envoyé un mail. Peut-être que les gens ne l'ont pas lu, mais (regardant l'assistant dans les yeux) ils sont tous d'accord n'est-ce pas? »

Médecin assistant : Ne répond pas, baisse les yeux.

Suit un rire gêné du médecin-cadre et un silence dans la salle, avant que la réunion ne reprenne son cours.

Après la séance, le médecin assistant nous expliquera qu'il refuse de signer un consentement « libre et éclairé » alors que sa participation à la réunion est obligatoire. Le recroisant quelques jours plus tard dans les couloirs du service, il nous expliquera que ce refus n'est en rien lié à notre recherche, mais qu'il exprime son mécontentement à l'égard de la hiérarchie. Il finira d'ailleurs par accepter de signer le formulaire de consentement. Si cet exemple montre une nouvelle fois l'importance de la négociation du consentement en amont, il indique également que les relations hiérarchiques et la rotation fréquente des médecins assistants (et du personnel en général), spécifiques à certaines institutions de santé, constituent des obstacles conséquents à cette précaution.

Faire signer le "consentement" aux patients et patientes : la gestion des normes et contraintes éthiques

Les enregistrements vidéo ne font pas, en soi, l'objet de dispositions particulières dans le droit suisse relatif à la recherche «sur l'être humain $»^{12}$. En revanche, le fait de récolter des données personnelles et médicales à propos des patients et patientes contraint à obtenir la validation du projet par une commission d'éthique dans le but, imposé par la Constitution, de protéger la «dignité » et la «personnalité » des individus. La loi précise dès lors que, parallèlement aux essais cliniques et à la collecte de matériel biologique, doit être approuvé par une commission d'éthique tout projet 
dans le cadre duquel des «données personnelles liées à [leur] santé sont collectées dans le but de répondre à une problématique scientifique ${ }^{13}$. Bien que pour nous les données personnelles et médicales des patients et patientes soient secondaires dans l'analyse, le fait de les enregistrer par vidéo, y compris lorsque nous filmons une réunion professionnelle lors de laquelle sont discutés leurs cas, distingue clairement notre démarche d'une observation directe. Une démarche de type ethnographique ne recourant pas à la vidéo peut être considérée comme ne devant pas faire l'objet d'une approbation par une commission d'éthique si le respect du secret professionnel et médical est garanti, dans la mesure où il est possible de ne pas prendre note des données médicales des patients et patientes ou de le faire de manière anonymisée. Cette remarque n'est bien sûr pas valable pour les pays au sein desquels les exigences éthiques sont plus élevées, en particulier dans le monde anglo-saxon et au Québec ${ }^{14}$, où celles-ci ne sont pas limitées à la protection des données médicales, mais concernent la recherche en sciences humaines et sociales de manière générale.

En ce qui nous concerne, la démarche éthique a consisté, une fois l'accord de la commission reçu, à obtenir celui des patientes et patients filmés et à leur faire signer un « consentement libre et éclairé » relatif à leur participation à l'étude. Classiquement, celui-ci consiste en une présentation de l'étude et de son déroulement; des informations sur les bénéfices, risques et contraintes pour le patient ou la patiente; d'autres informations à propos du respect de la confidentialité des données; les coordonnées d'une personne de contact. La règle veut que le consentement soit transmis aux patients et patientes quelques jours avant leur participation afin de leur laisser un temps de réflexion. Parallèlement au consentement général, nous avons également donné la possibilité à ceux qui se font filmer (ainsi d'ailleurs qu'aux professionnels et professionnelles) de donner leur accord sur des usages différenciés des images. Ainsi, pour les images elles-mêmes, d'une part, et pour leur transcription détaillée, d'autre part, les personnes filmées peuvent donner leur accord ou non (cases à cocher) pour un usage dans le cadre de formations, de présentations scientifiques et de publications (voir l'Encadré 2).

Cette possibilité de détailler le consentement allonge le temps nécessaire à la présentation des documents aux personnes que nous souhaitons filmer. Ce détail dans le consentement offre cependant une marge d'autonomie qui peut sans nul doute faciliter l'obtention de l'accord des personnes. Notre expérience montre cependant que ce sont davantage les professionnels et professionnelles que les patients et patientes qui ont refusé que nous fassions certains des usages énumérés, même si d'une manière générale, les personnes acceptant d'être filmées acceptent également toutes les modalités d'usage des images et des transcriptions. Notons que la récolte des consentements des patients et patientes est plus facile dans les petites institutions, ainsi que dans celles où ils restent plus longtemps. La récolte est en revanche rendue plus difficile lorsque les personnes présentent des troubles cognitifs ou sont mineures, car 
Le formulaire distribué aux deux groupes d'individus demande leur accord pour les usages suivants :

- Les enregistrements vidéo pourraient être utilisés pour former des $\square$ Oui professionnels et professionnelles. $\quad \square$ Non

- Les transcriptions pourraient être utilisées pour former des $\square$ Oui professionnels et professionnelles. $\quad \square$ Non

- Les enregistrements vidéo pourraient être utilisés pour des présentations $\square$ Oui scientifiques. $\quad \square$ Non

- Les transcriptions pourraient être utilisées pour des présentations $\square$ Oui scientifiques. $\quad \square$ Non

- Les transcriptions pourraient être utilisées dans des publications $\square$ Oui $\begin{array}{ll}\text { scientifiques. } & \square \text { Non }\end{array}$

Encadré 2. Consentement détaillé sur les usages des enregistrements et de leur transcription.

cela nécessite de prendre contact avec leurs proches ou leurs représentantes ou représentants légaux.

Il faut ajouter, finalement, que la validation par la commission d'éthique est un élément central pour négocier l'accès aux terrains d'observation. Si elle est garante de la protection de la dignité et de la personnalité des patients et patientes, elle renforce également la valeur scientifique de la recherche qualitative dans un contexte plus habitué aux recherches cliniques, ces dernières posant des problèmes et dilemmes éthiques souvent plus importants.

\section{Conclusion}

Souvent utilisée dans le cadre de recherches s'intéressant aux actions situées et aux logiques interactionnelles, la vidéo est pourtant un outil puissant de production de données largement mésestimé par les sciences sociales. Permettant de capter des situations naturelles dans toute leur densité et de les analyser ensuite en équipe, cet outil offrirait pourtant des opportunités scientifiques significatives à différents types de recherche portant sur les comportements sociaux, quelles que soient les approches ou perspectives privilégiées. La vidéo offre la possibilité, comme le dictaphone, d'enregistrer le langage verbal, tout en facilitant largement la transcription de discussions de groupe en permettant d'identifier les locuteurs. En parallèle, elle capte avec d'infinis détails les pratiques non langagières, la communication non verbale ainsi que l'usage des outils et les rapports entre humains et espace matériel. Combinée en 
particulier à une immersion et des observations ethnographiques, elle ouvre des horizons qu'il nous semble particulièrement pertinent d'explorer. Et ce, d'autant que, si les enregistrements vidéo obligent à effectuer des choix techniques et sont entourés d'enjeux éthiques spécifiques, faire accepter la présence de la caméra au sein des institutions de santé ne présente pas de difficultés significativement plus élevées que les négociations imposées par toute démarche ethnographique classique.

\section{Notes}

${ }^{1}$ Voir par exemple Becker, Geer, Hughes, \& Strauss (1977), et Strauss, Fagerhaugh, Suczek, \& Wiener (1985).

2 «All elements that constitute the meaning of an image are simultaneously present» (Raab \& Tänzler, 2012, p. 86). Notons cependant que, en tous cas dans l'état actuel des développements technologiques, la vidéo n'enregistre pas les odeurs.

3 «Video data is certainly among the most complex data in social scientific empirical research » (Knoblauch et al., 2012, p. 14).

${ }^{4}$ « that prioritises the situated and interactional accomplishment of practical action » (Heath et al., 2010, p. 1).

5 «structures and patterns of interaction, such as the coordination of work activities, the course of family arguments or professional meetings » (Knoblauch, 2012, p. 72).

${ }^{6}$ Même si, sur ce second point, la présence récurrente de stagiaires dans les institutions peut constituer un rôle disponible pour les chercheurs et chercheuses.

${ }^{7}$ Notre équipe, dont la plupart des membres sont formés aux sciences sociales, comprend plusieurs physiothérapeutes (exerçant ou non), une psychologue ainsi qu'une infirmière.

${ }^{8}$ Ce transfert de nouvelles connaissances dans la pratique professionnelle est inspiré de la Conversation-analytic role play method développée par Stokoe (2014).

${ }^{9}$ Les négociations ont pu alors porter sur la position des noms des différents auteurs de la publication pressentie.

${ }^{10}$ Nous restons volontairement flous sur les domaines médicaux concernés afin de garantir l'anonymat de ces services.

${ }^{11}$ Une délégation partielle puisque, si nos analyses sont transmises et discutées avec les acteurs de terrain, les données vidéo appartiennent exclusivement à l'équipe de recherche et ne sont transmises d'aucune manière, ni aux personnes filmées, ni à la direction.

${ }^{12}$ Voir la Loi fédérale relative à la recherche sur l'être humain (LRH) du 30 septembre 2011.

${ }^{13}$ Loi fédérale relative à la recherche sur l'être humain (LRH) du 30 septembre 2011.

${ }^{14}$ Voir par exemple les principes éthiques édictés par l'Economic and Social Research Council au Royaume-Uni ou, au Canada, le document officiel : Conseil de recherches en sciences humaines du Canada, Conseil de recherches en sciences naturelles et en génie du Canada, Instituts de recherche en santé du Canada (2014) Énoncé de politique des trois Conseils: Éthique de la recherche avec des êtres humains. 


\section{Références}

Avril, C., Cartier, M., \& Serre, D. (2010). Enquêter sur le travail. Concepts, méthodes, récits. Paris : La Découverte.

Beaud, S., \& Weber, F. (2010). Guide de l'enquête de terrain produire et analyser des données ethnographiques (4 éd.). Paris : La Découverte.

Becker, H. S., Geer, B., Hughes, E. C., \& Strauss, A. L. (1977). Boys in white. Student culture in medical school. New Brunswick, NJ : Transaction Books.

Clot, Y., Faïta, D., Fernandez, G., \& Scheller, L. (2000). Entretiens en autoconfrontation croisée: Une méthode en clinique de l'activité. PISTES Perspectives interdisciplinaires sur le travail et la santé, 2(1), 1-9.

Darmon, M. (2005). Le psychiatre, la sociologue et la boulangère. Analyse d'un refus de terrain. Genèses, 1(58), 98-112.

Dubar, C., Tripier, P., \& Boussard, V. (2011). Sociologie des professions. Paris: Armand Colin.

Foley, R.-A. (2016). L'observation. Dans J. Kivits, F. Balard, C. Fournier, \& M. Winance (Éds), Les recherches qualitatives en santé (pp. 117-133). Malakoff : Armand Colin.

Heath, C., Hindmarsh, J., \& Luff, P. (2010). Video in qualitative research. Analysing social interaction in everyday life. Los Angeles, CA : Sage.

Hindmarsh, J., \& Pilnick, A. (2007). Knowing bodies at work. Embodiment and ephemeral teamwork in anaesthesia. Organization Studies, 28(9), 1395-1416.

Hughes, E. C. (1958). Men and their work. Glencoe : The Free Press.

Keel, S., \& Schoeb, V. (2016). Professionals' embodied orientations towards patients in discharge-planning meetings and their impact on patient participation. Communication \& Medicine, 13(1), 115-134.

Knoblauch, H. (2012). Videography. Focused ethnography and video analysis. Dans H. Knoblauch, H.-G. Soeffner, J. Raab, \& B. Schnettler (Éds), Video analysis : Methodology and methods. Qualitative audiovisual data analysis in sociology (pp. 69-83). Francfort-sur-le-Main : P. Lang.

Knoblauch, H., Soeffner, H.-G., Raab, J., \& Schnettler, B. (Éds). (2012). Video analysis: Methodology and methods. Qualitative audiovisual data analysis in sociology. Francfort-sur-le-Main : P. Lang.

Koschmann, T., LeBaron, C., Goodwin, C., Zemel, A., \& Dunnington, G. (2007). Formulating the triangle of doom. Gesture, 7(1), 97-118. 
Lambelet, A. (2010). Analyser les rassemblements au moyen de photographies ou de films. Pistes et enjeux. ethnographiques.org, 21. Repéré à http://www.ethnographiques.org/spip.php?article712

Luckmann, T. (2012). Some remarks on scores in multimodal sequential analysis. Dans H. Knoblauch, H.-G. Soeffner, J. Raab, \& B. Schnettler (Éds), Video analysis : Methodology and methods. Qualitative audiovisual data analysis in sociology (pp. 29-34). Francfort-sur-le-Main : P. Lang.

Luff, P., Hindmarsh, J., \& Heath, C. (Éds). (2000). Workplace studies. Recovering work practice and informing system design. Cambridge : Cambridge University Press.

Mondada, L. (2014). Instructions in the operating room. How the surgeon directs their assistant's hands. Discourse Studies, 16(2), 131-161.

Parry, R. (2010). Video-based conversation analysis. Dans I. L. Bourgeault, R. Dingwall, \& R. G. De Vries (Éds), The Sage handbook of qualitative methods in health research (pp. 373-396). Los Angeles, CA : Sage.

Raab, J., \& Tänzler, D. (2012). Video hermeneutics. Dans H. Knoblauch, H.-G. Soeffner, J. Raab, \& B. Schnettler (Éds), Video analysis: Methodology and methods. Qualitative audiovisual data analysis in sociology (pp. 85-97). Francfortsur-le-Main : P. Lang.

Robinson, J. D. (1998). Getting down to business talk, gaze, and body orientation during openings of doctor-patient consultations. Human Communication Research, 25(1), 97-123.

Ruusuvuori, J. (2001). Looking means listening. Coordinating displays of engagement in doctor-patient interaction. Social Science \& Medicine, 52(7), 1093-1108.

Schoeb, V., Hartmeier, A., \& Keel, S. (2015). Transfer von Forschungsresultaten zur Austrittsplanung in drei Schweizer Rehabilitationskliniken. Reflektion als Sprungbrett für Veränderungen im Praxisalltag [Transfert des résultats de recherche à propos de la décision de sortie dans trois institutions de réhabilitation en Suisse. La réflexion comme tremplin pour le changement dans les pratiques quotidiennes]. Schweizerische Ärztezeitung, 5(96), 132-136.

Schubert, C. (2012). Video analysis of practice and the practice of video analysis. Selecting field and focus in videography. Dans H. Knoblauch, H.-G. Soeffner, J. Raab, \& B. Schnettler (Éds), Video analysis: Methodology and methods. Qualitative audiovisual data analysis in sociology (pp. 115-126). Francfort-sur-leMain : P. Lang. 
Stokoe, E. (2014). The conversation analytic role-play method (CARM). A method for training communication skills as an alternative to simulated role-play. Research on Language and Social Interaction, 47(3), 255-265.

Strauss, A. L., Fagerhaugh, S., Suczek, B., \& Wiener, C. (1985). Social organization of medical work. Chicago, IL : The University of Chicago Press.

Tiitinen, S., \& Ruusuvuori, J. (2012). Engaging parents through gaze. Speaker selection in three-party interactions in maternity clinics. Patient Education and Counseling, 89(1), 38-43.

David Pichonnaz est docteur en sociologie (Université de Fribourg, Suisse, et École des hautes études en sciences sociales, Paris). Il est chercheur à la Haute école de santé Vaud et enseignant à l'Université de Lausanne (Suisse). Spécialisé dans l'étude du travail et des groupes professionnels, il s'intéresse notamment à la collaboration interprofessionnelle dans le monde de la santé et à la socialisation aux métiers relationnels. Ses travaux sont principalement basés sur des données qualitatives et sur une démarche de type ethnographique.

Camille Bécherraz est titulaire d'un Master en sciences sociales. Elle est assistante d'enseignement et de recherche à la Haute école de santé Vaud, à Lausanne (Suisse). Ses travaux de recherche s'intéressent à la relation en santé ainsi qu'à l'éducation interprofessionnelle et sont ancrés dans une démarche à la fois qualitative et quantitative. Elle enseigne la communication en santé et la collaboration interprofessionnelle.

Isabelle Knutti est titulaire d'un Master en physiothérapie, profession qu'elle exerce comme indépendante. Elle est en outre assistante de recherche à la Haute école de santé Vaud, à Lausanne (Suisse), où elle participe à la conduite d'un projet de recherche consacré à la collaboration interprofessionnelle.

Liliane Staffoni est titulaire d'un Bachelor en physiothérapie et d'un Master en psychologie. Professeure associée à la Haute école de santé Vaud, à Lausanne (Suisse), elle est la requérante principale d'un projet financé par le Fonds national suisse de la recherche scientifique consacré à l'interprofessionnalité dans les soins. Ses domaines d'intérêt sont la psychologie de la santé et du développement ainsi que l'interprofessionnalité dans les soins.

Veronika Schoeb est docteure en sociologie (Université de Nottingham, Royaume-Uni). Elle est professeure assistante au Department of Rehabilitation Sciences de la Hong Kong Polytechnic University (Chine) et adjointe scientifique à la Haute école de santé Vaud, à Lausanne (Suisse). Elle a obtenu plusieurs financements de recherche en Suisse et à l'étranger afin d'étudier, selon une approche qualitative, les interactions entre patients et professionnels de santé, ainsi qu'au sein des équipes interprofessionnelles. 